\title{
Modified 9DLT Matrix for Similarity Retrieval of Line-Drawing Images
}

\author{
Naveen Onkarappa* and D.S. Guru \\ Department of Studies in Computer Science, University of Mysore, \\ Mysore - 570 006, India \\ naveen_msc@yahoo.com, dsg@compsci.uni-mysore.ac.in
}

\begin{abstract}
An attempt towards perception of spatial relationships existing among the generic components of line-drawing images is made for their similarity retrieval. The proposed work is based on modified 9DLT matrix representation. The conventional concept of 9DLT matrix has been tuned to accommodate multiple occurrences of identical components in images. A novel similarity measure to estimate the degree of similarity between two 9DLT matrices representing images is introduced and exploited for retrieval of line-drawing images. A database of 118 line-drawing images has been created to corroborate the effectiveness of the proposed model for similarity retrieval through an extensive experimentation.
\end{abstract}

\section{Introduction}

As the availability of information in the form of images is increasing, it has become important to process, store and search the images based on their content. Here in this paper, we concentrate on the need of storing and searching of line-drawing images (scanned copies of paper based drawings). Generally, line-drawing images (which include flowcharts, flow diagrams and engineering drawings such as electrical circuit diagrams, logic circuit diagrams and architectural plans) are made up of primitive components such as straight lines, curve segments, geometrical shapes and symbols. The possible dispersion of primitive components leads to many different line-drawings. The crucial feature that we can make out in discriminating line-drawing images is thus the spatial locations of the primitive components with respect to the other primitive components. In literature, few approaches for recognizing [18], matching [6]16], and interpreting [15] of line-drawing images have been proposed. Though Fanti et al., (2000) consider the spatial knowledge globally, it cannot discriminate line-drawing images considering the specific spatial relationships between specific components.

Thus an attempt to exploit the spatial knowledge, an important feature in discriminating images, for the purpose of similarity retrieval of line-drawing images is made in this paper. There have been several theoretical works towards development of effective spatial data structures, viz., nine-directional codes [129], triangular spatial relationship [8], spatial orientation graph [7] and 2D-strings and its variants

\footnotetext{
^ Corresponding author.
} 
[13 4 13 17]. Chang and Wu (1995) have proposed a technique for exact match retrieval based on 9DLT matrix [2] using principal component analysis. A survey of indexing and retrieval of iconic images can be found in [14. The similarity retrieval 1112 using 2D-strings and its variants involve subsequence matching which is non-deterministic polynomial complexity problem. Most of the previous representation and retrieval methods based on variants of nine-directional codes and 2Dstring representations are limited by the number/type of components (primitive objects) in the database, in addition to being ineffectual in handling multiple similar spatial relationships between identical components in images.

In this paper, a similarity retrieval of line-drawing images from a database using modified 9DLT matrix representation is proposed. The conventional 9DLT matrix which supports a single existence of a component and exact match retrieval is tuned up to accommodate multiple components/spatial relationships and also to support similarity retrieval. The proposed similarity measure is demonstrated on a database of 118 synthetic line-drawing images containing primitive components such as lines, circles, triangles, rectangles and trapeziums. The overall contributions of the paper are: 1. Modification of 9DLT matrix to accommodate multiple occurrences of identical components/spatial relationships, 2. A novel similarity measure to estimate the similarity between two such 9DLT matrices, and 3. Exploitation of spatial topology for similarity retrieval of line-drawing images.

This paper is organized as follows. Section 2 gives an overview of 9DLT matrix representation of symbolic images. In section 3, proposed transformation of physical line-drawing images into symbolic representation and the corresponding retrieval schemes are presented. Section 4 shows the retrieval results of the proposed scheme on a database of 118 images. Finally section 5 follows with conclusion.

\section{9DLT Matrix: An Overview}

Consider an abstract image consisting of four components with labels $L_{1}, L_{2}$, $L_{3}$ and $L_{4}$ as shown in Fig.1(a). We may use nine directional codes shown in Fig.1(b) to represent the pair-wise spatial relationships between $x$, a referenced component and $y$, a contrasted component. The directional code say $d=0$, represents that $y$ is to the east of $x, d=1$ represents that $y$ is to the north-east of $x$, and so on. Thus, the 9DLT matrix $T$ for the image of Fig.1(a) is as shown in Fig.1(c). Since each relationship is represented by a single triple $(x, y, d)$, the 9DLT matrix is a lower triangular matrix.

The 9DLT matrix can now be formally defined as follows (Chang, 1991). Let $V=\left\{v_{1}, v_{2}, v_{3}, v_{4}, \cdots, v_{m}\right\}$ be a set of $m$ distinct components/objects. Let $Z$ consist of ordered components $z_{1}, z_{2}, z_{3}, z_{s}$ such that, $\forall i=1,2, \cdots, s, z_{i} \in V$. Let $C$ be the set of nine-directional codes as defined in Fig.1(b). Each directional code is used to specify the spatial relationship between two components. So, a 9DLT matrix $T$ is an $s \times s$ matrix over $C$ in which $t_{i j}$, the $i^{\text {th }}$ row and $j^{\text {th }}$ column element of $T$ is the directional code of $Z_{j}$ to $Z_{i}$ if $j<i$, and undefined otherwise. The matrix $T$ is a 9DLT matrix according to the ordered set $Z$. 


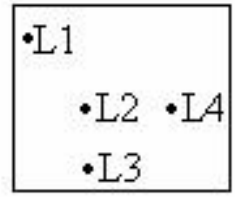

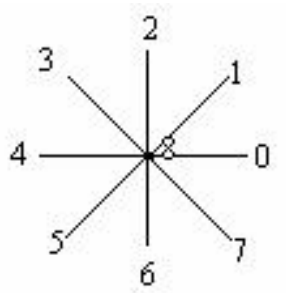

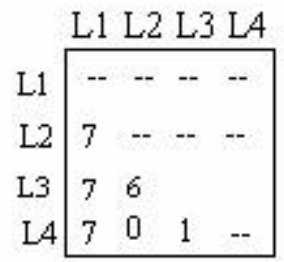

Fig. 1. (a):An abstract image, (b):The nine directional codes and (c):The 9DLT matrix of Fig.1(a)

\section{Proposed Scheme}

In this section, the method of transforming physical line-drawing images into their symbolic representation is presented. The symbolic representation is done using nine-directional spatial relationships. Using this representation, a scheme for retrieval of similar images is also proposed.

\subsection{Symbolic Representation of Line-Drawing Images}

The technique of measuring similarity between two images requires the images to be represented in some suitable form. Therefore it is necessary to transform the line-drawing images into symbolic form. In the transformation procedure, we first detect the straight lines using hough transform [5]. Using these detected straight lines, we find the possibility of constructing triangles, trapeziums and rectangles, and extract those possible constructs. After constructing the possible triangles, trapeziums and rectangles, the remaining non-contributing straight lines are generalized as line components. We also extract the circles present in the images using hough transform [10]. Using these extracted components (circles, triangles, trapeziums, rectangles and remaining straight lines) in an image, 9DLT matrices are constructed by considering components of particular type one after the other in a predefined sequence. That is, all line components first, followed by circles, rectangles, triangles and trapeziums. This constraint simplifies the similarity measure presented in section 3.2. While determining directional codes we are considering the area of components rather than just points (centroids) of components because such a representation is too sensitive in spatial reasoning. This is to decide the directional code in case of one component spreads over many directions with respect to another component. In such situations, the directional code is determined as the direction, where the maximum amount of referring component lies with respect to the other component. Further the triplets are generated for each 9DLT matrix corresponding to an image in the database. To generate the triplets, the components of particular type are given unique label. Each triplet of the form $\left(L_{1}, L_{2}, d\right)$ indicates that the component with label $L_{1}$ is in $d$-direction to the component with label $L_{2}$. Thus the physical line-drawing 
images are converted to symbolic representation as sets of triplets. Fig.2(c) shows the 9DLT matrix for the line-drawing image shown in Fig.2(a) using the directional codes in Fig 1. Fig. 2(b) shows the different components identified in Fig.2(a), where $L_{i}$ 's represent line components, $C_{i}$ 's represent circle components and $R_{i}$ 's represent rectangle components. By giving the unique labels to the components of particular type (here say label 10 for lines, label 20 for circles, label 30 for rectangles), the set of triplets generated for the image in Fig.2(a) is $S=\{(10,10,0),(10,10,2),(10,10,3),(10,10,1),(10,10,2),(10,10,7),(20,10,1)$, $(20,10,3),(20,10,0),(20,10,3),(30,10,2),(30,10,2),(30,10,7),(30,10,4),(30,20,6)\}$. This set of triplets generated for a line drawing image is then stored in the database as a representative of the image.
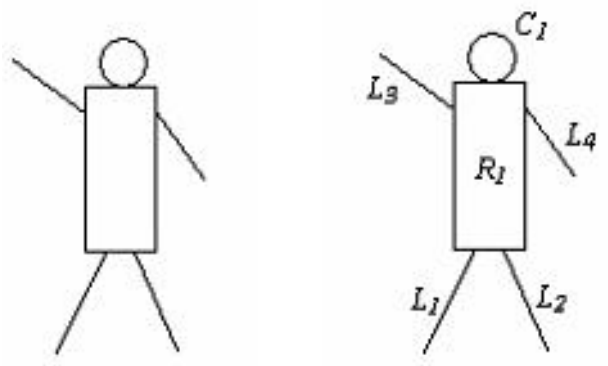

\begin{tabular}{|c|c|c|c|c|c|c|}
\hline & $L_{I}$ & $L_{2}$ & $L_{3}$ & $L_{4}$ & $C_{1}$ & $R_{I}$ \\
\hline$L_{1}$ & -- & -- & -- & -- & -- & -- \\
\hline$L_{2}$ & 0 & -- & -- & -- & -- & -- \\
\hline$L_{3}$ & 2 & 3 & -- & -- & -- & -- \\
\hline$L_{4}$ & 1 & 2 & 7 & -- & -- & -- \\
\hline$C_{2}$ & 1 & 3 & 0 & 3 & -- & - \\
\hline$R_{3}$ & 2 & 2 & 7 & 4 & 6 & -- \\
\hline
\end{tabular}

Fig. 2. (a). A line drawing image, 2(b). A labeled image of 2(a) and 2(c).Symbolic representation $2(\mathrm{a})$.

Thus, the following is the algorithm designed for creating a symbolic database of line-drawing images.

Algorithm 1: Symbolic database creation.

Input: Line Drawing Images.

Output: Symbolic representation of all line-drawing images.

Method:

Repeat the following steps for each input line-drawing image.

1. Detect straight lines and circles using hough transform.

2. Extract possible constructs (rectangles, trapeziums and triangles) using the detected straight lines as explained in section 3.1.

3. Using the extracted components (lines, circles, rectangles, triangles and trapeziums), construct the 9DLT matrix by considering components of particular type one after the other (i.e., all line components first, followed by circles, rectangles, triangles and trapeziums).

4. Construct the set of triplets for the 9DLT matrix by giving a unique label for a particular type of component and store it as a representative.

\section{Algorithm Ends}




\subsection{Similarity Retrieval}

The similarity between two line-drawing images (i.e., between two symbolic representations) is obtained in terms of the number of triplets in common between the representative sets of triplets. In finding the number of triplets in common, the uniqueness of two triplets is defined as follows.

Let $P_{1}=\left(L_{i 1}, L_{i 2}, d_{i}\right)$ and $P_{2}=\left(L_{j 1}, L_{j 2}, d_{j}\right)$ be two triplets. The two triplets $P_{1}$ and $P_{2}$ are same if $\left(\left(L_{i 1}==L_{j 1}\right)\right.$ and $\left(L_{i 2}==L_{j 2}\right)$ and further $\left.\left(d_{i}==d_{j}\right)\right)$ in the case $\left(L_{i 1} \neq L_{i 2}\right)$. In the case $\left(L_{i 1}==L_{i 2}\right)$, i.e., while comparing spatial relationships between particular type components, in addition to the conditions $\left(L_{i 1}==L_{j 1}\right)$ and $\left(L_{i 2}==L_{j 2}\right)$, it needs to be checked that $\left(d_{i}==d_{j}\right)$ or $d_{i}$ is oppositional (complementary) directional code of $d_{j}$. This is necessary because the components would have interchanged during the process of grouping and putting the components of particular type together at the time of 9DLT matrix construction. This complimentary directional check is not required in the case $\left(L_{i 1} \neq L_{i 2}\right)$, because there is no chance of interchange in components of different types due to the constraint of grouping and listing out them in a predefined sequence while constructing 9DLT matrix.

After finding out the number of triplets in common between two sets of triplets corresponding to two images (query and a reference image), we propose to compute the similarity between a query and reference image as follows.

$$
\text { Sim }=\frac{2 \times \text { number of triplets in common between query and reference image }}{\text { number of triplets in query }+ \text { number of triplets in reference }}
$$

The above proposed similarity measure ensures that the value of Sim lies in the range $[0,1]$. The following algorithm is thus devised to retrieve similar images for a given line-drawing query image.
Algorithm 2: Retrieval Scheme.
Input: $Q$, a query line-drawing image.
Output:List of line-drawing images.
Method:

1. Obtain the symbolic representation for a given line-drawing query image using algorithm 1.

2. Compute the similarity of the query image with each of the image in the database using equation 1.

3. Retrieve the images in the database in descending order of their similarities.

\section{Algorithm Ends}

Since the similarity between two line-drawing images is found using the number of triplets in common, the time complexity is of $O(m n)$, where $m$, is the number of triplets in query and $n$ is the number of triplets in referenced image. Thus the proposed methodology is computationally efficient when compared to exponential/non-polynomial computational times of few methods proposed in literature 314. 


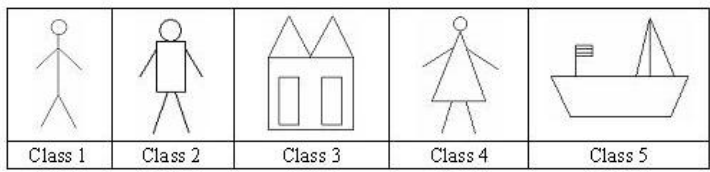

Fig. 3. Representative images of the five different classes

\begin{tabular}{|c|c|}
\hline Query image & $\begin{array}{c}\text { Retrieved images for the query } \\
\text { with similarity }=1\end{array}$ \\
\hline $\begin{array}{c}\text { Retrieved images for the query } \\
\text { with similarity }=0.9333\end{array}$ \\
\hline $\begin{array}{c}\text { Retrieved images for the query } \\
\text { with similarity }=0.8\end{array}$ \\
\hline $\begin{array}{c}\text { Retrieved images for the query } \\
\text { with similarity }=0.5714\end{array}$ \\
\hline
\end{tabular}

Fig. 4. Retrieval results for a query image of class 1

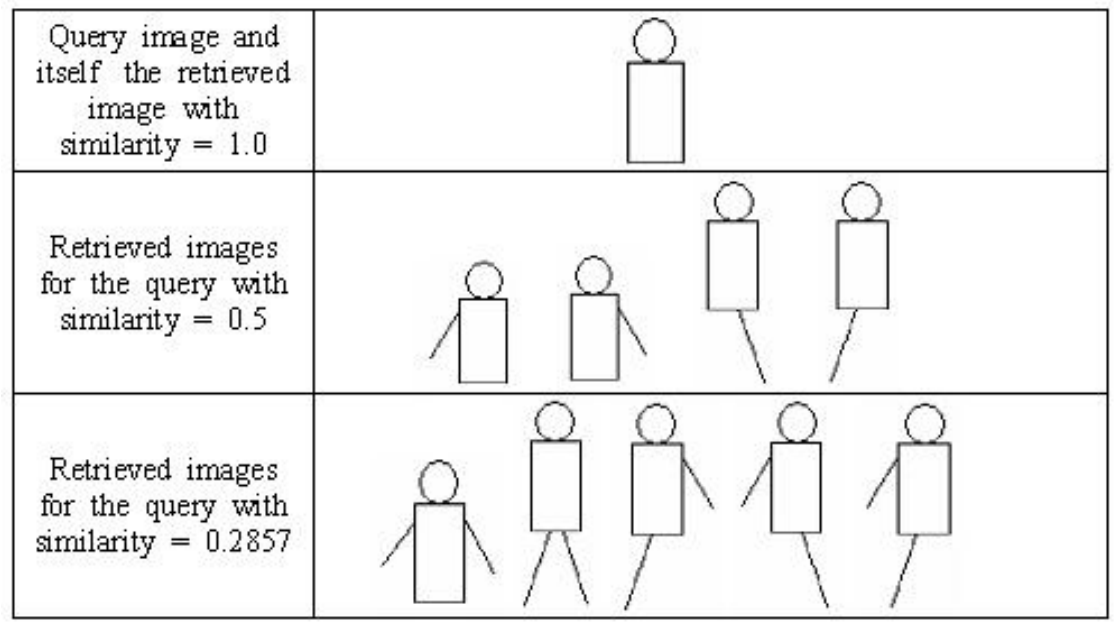

Fig. 5. Retrieval results for a query image of class 2 


\section{Experimental Results}

In order to validate the proposed representation and retrieval algorithms, we have created a database of 118 synthetic line-drawing images, representatives of which are shown in Fig.3. Out of the results obtained for many query images during experimentation, the retrieval results for only three query images are shown in Fig.4, Fig.5 and Fig.6. The top ten retrieved images with their similarity value for the two query images are given in Fig.4 and Fig.5. Fig.6 shows only four images retrieved for the query as our database contains only four images of that class. The experimental results on this database appear to be effectual and encouraging.

\begin{tabular}{|c|c|}
\hline $\begin{array}{c}\text { Query image and itself the retrieved } \\
\text { image with similarity }=1.0\end{array}$ & \\
\hline $\begin{array}{c}\text { Retrieved images for the query } \\
\text { with similarity }=0.2857\end{array}$ & \\
$\begin{array}{c}\text { Retrieved images for the query } \\
\text { with similarity }=0.1818\end{array}$
\end{tabular}

Fig. 6. Retrieval results for a query image of class 3

\section{Conclusion}

A similarity measure for retrieval of line-drawing images based on the modified 9DLT matrix representation is proposed. The proposed similarity retrieval scheme is invariant to translation, scale and can deal with multiple instances of components/relationships in images. The proposed method is validated on a database of 118 synthetic line-drawing images. The method can be extended to accommodate other primitives including curve segments. The focus is on extending the approach to automatic understanding and also towards indexing for efficient retrieval.

\section{References}

1. Chang, C.C., Wu, T.C.: An exact match retrieval scheme based upon principal component analysis. Pattern Recognition Letters 16(5), 465-470 (1995)

2. Chang, C.C.: Spatial Match Retrieval of Symbolic Pictures. J. Information Science and Engineering 7 17(3), 405-422 (1991) 
3. Chang, S.K., Shi, Q.Y., Yan, C.W.: Iconic Indexing by 2D Strings. IEEE Tran. on Pattern Analysis and Machine Intelligence 9(3), 413-428 (1987)

4. Chang, S.K., Yan, C.W., Dimitroff, D.C., Arndt, T.: An Intelligent Image Database System. IEEE Tran. Software Engineering 14(3) (1988)

5. Duda, R.O., Hart, P.E.: Use of the Hough Transformation to Detect Lines and Curves in Pictures. Comm. ACM 15, 11-15 (1972)

6. Franti, P., Mednonogov, A., Kalviainen, H.: Hough transform for rotation invariant matching of line-drawing images. In: Proceedings of the International Conf. on Pattern Recognition (ICPR), pp. 389-392 (2000)

7. Gudivada, V.N., Raghavan, V.V.: Design and Evaluation of Algorithms for Image Retrieval by Spatial Similarity. ACM Transactions on Information Systems 13(2), 115-144 (1995)

8. Guru, D.S., Nagabhushan, P.: Triangular spatial relationship: a new approach for spatial knowledge representation. Pattern Recognition Letters 22(9), 999-1006 (2001)

9. Po-Whei, H., Chu-Hui, L.: Image Database Design Based on 9D-SPA Representation for Spatial Relations. IEEE Transactions on Knowledge and Data Engineering 16(12), 1486-1496 (2004)

10. Kerbyson, D.J., Atherton, T.J.: Circle detection using Hough transform filters. In: Proceedings of Fifth International Conf. on Image Processing and its Applications, pp. 370-374 (1995)

11. Lee, S.Y., Hsu, F.J.: Spatial reasoning and similarity retrieval images using 2D-C string knowledge representation. Pattern Recognition 25(3), 305-318 (1992)

12. Lee, S.Y., Shan, M.K., Yang, W.P.: Similarity retrieval of iconic image database. Pattern Recognition 22(6), 675-682 (1989)

13. Lee, S.Y., Hsu, F.J.: 2D C-String: A New Spatial Knowledge Representation for Image Database Systems. Pattern Recognition 23(10), 1077-1087 (1990)

14. Punitha, P.: IARS: Image Archival and Retrieval Systems, Ph. D. Thesis, Dept. of Studies in Computer Science, University of Mysore, India (2006)

15. Kasturi, R., Bow, S.T., El-Masri, W., Shah, J., Gattiker, J.R., Mokate, U.B.: A System for Interpretation of Line Drawings. IEEE Tran. on Pattern Analysis and Machine Intelligence 12(10), 978-992 (1990)

16. Tabbone, S., Wendling, L., Tombre, K.: Matching of graphical symbols in linedrawing images using angular signature information. International Journal on Document Analysis and Recognition 6(2), 115-125 (2003)

17. Ying-Hong, W.: Image indexing and similarity retrieval based on spatial relationship model. Information Sciences 154, 39-58 (2003)

18. Yu, Y., Samal, A., Seth, S.C.: A System for Recognizing a Large Class of Engineering Drawings. IEEE Tran. on Pattern Analysis and Machine Intelligence 19(8), 868-890 (1997) 